$$
\text { CONF- } 9606226--13 \quad \text { GA-A22406 }
$$

\title{
MODELING OF IMPURITY SPECTROSCOPY IN THE DIVERTOR AND SOL OF DIII-D USING THE 1D MULLTIFLUID MODEL NEWT1D
}

by
W.P. WEST, T.E. EVANS, N.H. BROOKS, M.E. FENSTERMACHER,
S. HIRSHMAN, R.C. ISLER, A.W. LEONARD, G.D. PORTER,
M.R. WADE, D.G. WHYTE, and R.D. WOOD,

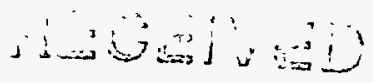

MPAR 171997

(10) 91

MISTRIITION OF THIS DOCUMENT IS UNLIMITED

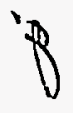




\section{MODELING OF IMPURITY SPECTROSCOPY IN THE DIVERTOR AND SOL OF DIII-D USING THE 1D MULLTIFLUID MODEL NEWT1D}

\section{by}

W.P. WEST, T.E. EVANS, N.H. BROOKS, M.E. FENSTERMACHER, $\dagger$ S. HIRSHMAN, $¥$ R.C. ISLER, $¥$ A.W. LEONARD, G.D. PORTER, $†$ M.R. WADE, $¥$ D.G. WHYTE, $\Delta$ and R.D. WOOD, ${ }^{\dagger}$

This is a preprint of a paper to be presented at the Twenty-Third European Conference on Controlled Fusion and Plasma Physics, June 24-28, 1996, Kiev, Ukraine, and to be published in the Proceedings.

$\dagger_{\text {Lawrence Livermore National Laboratory }}$ Ғoak Ridge National Laboratory

$\triangle$ INRS-Energie et Materiaux

Work supported by the U.S. Department of Energy under Contract Nos. DE-AC03-89ER51114, W-7405-ENG-48, and DE-AC05-96OR22464 


\section{DISCLAIMER}

Portions of this document may be illegible in electronic image products. Images are produced from the best available original document. 


\title{
MODELING OF IMPURITY SPECTROSCOPY IN THE DIVERTOR AND SOL OF DIII-D USING THE 1D MULTIFLUID MODEL NEWT1D*
}

\author{
W.P. West, T.E. Evans, N.H. Brooks, M.E. Fenstermacher, $\dagger^{\dagger}$ S. Hirshman $\ddagger$ R.C. Isler $\ddagger$ \\ A.W. Leonard, G.D. Porter, ${ }^{\dagger}$ M.R. Wade, $\ddagger$ D.G. Whyte, ${ }^{\Delta}$ R.D. Wood ${ }^{\dagger}$ \\ General Atomics, P.O. Box 85608, San Diego, California 92186-9784 U.S.A.
}

NEWT1D, a one dimensional multifluid model of the scrape-off layer and divertor plasma, has been used to model the plasma including the distribution of carbon ionization states in the SOL and divertor of ELMing $\mathrm{H}$-mode at two injected power levels in DII-D. Comparison of the code predictions to the measured divertor and scrape-off layer (SOL) plasma density and temperature shows good agreement. Comparison of the predicted line emissions to the spectroscopic data suggests that physically sputtered carbon from the strike point is not transported up the flux tube; a distributed source of carbon a few centemeters up the flux tube is required to achieve reasonable agreement.

\section{Introduction}

A basic understanding of the tokamak divertor, including impurity transport, is required in order to develop predictive capability for future high power tokamak divertors. However the strong spatial gradients in the divertor and SOL plasmas make detailed understanding difficult, both theoretically and experimentally. Benchmarking the complex codes used to model the divertor plasma against data from present day diverted tokamaks is key to assuring a detailed understanding of the plasma transport and to providing predictive capability. To date, 2D modeling codes that include impurities require long run times on even the fastest workstations, and therefore are not easily used for analysis of experimental results. The relatively simplistic approach of a 1D model has obvious shortcomings, failing to address rigorously radial plasma transport and the inherently 2D (or even 3D) problem of neutral transport. However the NEWT1D multifluid code handles impurities implicitly, and converges quickly. Here we have used the 1D modeling of impurity spectroscopy to yield some insight into the distribution of carbon ions in the divertor. The model provides detailed information on both the plasma parameters and impurity ionization state distribution along the flux tube just outside the seperatrix, where the plasma temperature and density is highest in the divertor. The plasma parameters and calculated impurity line emissions can be compared directly to DIII-D data.

The discharges used in this work are a part of recent experiments on DIII-D focusing on characterizing the divertor and SOL plasma in great detail using divertor and midplane Thomson scattering, fixed and plunging Langmuir probes, bolometry, IR cameras, and visible and VUV spectroscopy. ${ }^{1}$ Here, the data from a set of attached ELMing $\mathrm{H}$-mode discharges at two injected beam power levels, 3.1 MW, and 8.4 MW, are compared to the output of the NEWT1D model. Matching the poloidal distributions of plasma electron density and temperature and the midplane values of electron density and temperature and ion temperature gives confidence that the parallel impurity transport (dominated by flow friction, ion temperature gradient force, and pressure gradients) simulated in the code is representative of the transport expected in these plasmas.

\section{The NEWT1D Model}

The NEWT1D code is a multifluid model of a single open field line flux tube from the midplane to strike plate of a diverted tokamak. The model includes the momentum and energy balance between the electron fluid and the ion fluids; impurity and neutral radiation is treated as a loss term for the electron energy balance. Particle and momentum balance are treated independently for the primary ion and each impurity ionization state, however all ion fluids are assumed to be at the same temperature. The code is capable of modeling up to three impurity species simultaneously; in this work only carbon has been studied. A choice of parallel transport models is available, either a Braginskii model ${ }^{2}$ or the Hirshman-Sigmar formulation. ${ }^{3}$ In this study, only the Hirshman-Sigmar model has been used. The magnetic geometry of the flux tubes are obtained from the EFIT magnetic equilibrium reconstruction code.

\footnotetext{
*Work supported by U.S. Department of Energy under Contract Nos. DE-AC03-89ER51114, DE-AC05-900R22464, and W-7405-ENG-48.

†Lawrence Livermore National Laboratory.

†Oak Ridge National Laboratory.

$\triangle$ INRS-Energie et Materiaux.
} 
The primary inputs to the code are: (1) power (ion and electron) and particle fluxes from the core plasma into the flux tube, (2) primary ion recycling coefficent, (3) impurity recycling coefficient (= zero), (4) a multiplier for impurity sputtering temperature dependence (the impurity sputtering rate is adjusted according to the electron temperature at the plate) was kept fixed at 0.01 , (5) impurity gas puffing along the flux tube as described below. Perpendicular transport is included as an ad hoc loss term, and is specified in the input by transport coefficients and a radial scale length. The midplane end of the flux tube is a stagnation point. At the strike plate, all ion fluid velocities are assumed equal to the primary ion sonic velocity. The ionization, recombination, and total radiation rates are obtained from the ADPAK data base. ${ }^{4}$

Modeling the spectroscopic data is done in a post-processing mode. The impurity ion density and excitation rate distributions along the flux tube are mapped onto the geometry of the spectrometer viewing chords on DIII-D and line integrated emissivities are calculated. The excitation rates at the electron densities and temperatures along the flux tube are obtained from a collisional-radiative model of the CII, CIII, and CIV ions. ${ }^{5}$ In these discharges the dominant impurity is carbon, being the order of $2 \%$ of the electron density. In the model, the carbon sources, sputtering at the strike plate and neutral injection at selected locations along the flux tube, are adjusted until reasonable agreement is obtained with the visible and VUV spectroscopic data.

\section{The Experimental Data}

The DIII-D tokamak, with an extenisve set of divertor diagnostics and a flexible and dynamic plasma shape control system, is suited to detailed characterization of the 2D structure of the divertor plasma. ${ }^{1}$ Diagnostic chords of divertor Thomson scattering and visible and VUV spectrometers pass through a central region of the divertor floor, and the $\mathrm{X}$-point and strike points are swept past these chords as a function of time during the discharge. This sweep also provides a detailed picture of the electron density and temperature at the strike points as they are swept past fixed position domed probes. At the midplane detailed measurements of the electron density and temperature are obtained with the core Thomson scattering system and ion temperatures with the Charge Exchange Recombination Spectrscopy (CER) system. All of these data are mapped onto the flux tube geometry using the EFIT magnetic reconstruction code.

The VUV emissivity data from 100 to $1100 \AA$ is obtained with an absolutely calibrated vertically viewing SPRED spectrometer, having a viewing width of $8 \mathrm{~cm}$ at the floor of DIII-D. ${ }^{7}$ An integration time of $50 \mathrm{~ms}$ was used. In all of the data shown, the variation due to the ELM's has been included in the averaging.

\section{Comparison of the Model to the Data}

The measured and calculated electron density and temperature as a function of normalized poloidal length from the strike plate, over the region of the outer divertor leg, is shown (Fig. 1) for both the high and low injected power cases. Some of the important discharge characteristics and the model input parameters are in Table 1.

\begin{tabular}{|c|c|c|}
\hline Plasma Parameters & High Power & Low Power \\
\hline $\begin{array}{l}\text { Injected Beam, Total Radiated, Dvertor Radiated Power (MW) } \\
\text { Ip (MA), g95 } \\
<n_{e}>\left(m^{-3}\right) \\
\text { Code Inputs: }\end{array}$ & $\begin{array}{c}8.4,4.2,2.8 \\
1.4,4.8 \\
6.2 \times 10^{19}\end{array}$ & $\begin{array}{c}3.1,1.6,1.0 \\
1.4,4.5 \\
7.0 \times 10^{19}\end{array}$ \\
\hline $\begin{array}{l}\text { Electron lon Input Power (MW) } \\
\mathrm{D}^{+} \text {Particle Input }\left(\mathrm{s}^{-1}\right) \text {, Recycling Coefficient } \\
\text { Carbon Sputtering Coefficient (multiplier) } \\
\text { Neutral Carbon Injection Rate }\left(\mathrm{s}^{-1}\right)\end{array}$ & $\begin{array}{l}1.96,2.94 \\
1.1 \times 10^{22}, 0.987 \\
0.003(0.01) \\
1.2 \times 10^{20}\end{array}$ & $\begin{array}{c}0.25,1.60 \\
8.0 \times 10^{21} 0.987 \\
0.002(0.01) \\
7.5 \times 10^{19}\end{array}$ \\
\hline
\end{tabular}

To compare the calculated electron densities and temperatures along the outer divertor leg, the Thomson scattering points are mapped onto $\left(\psi_{\text {norm }}=\psi-\psi_{0} / \psi_{\text {sep }}-\psi_{0}\right)$ and $L_{\text {norm }}=$ poloidal length from platel

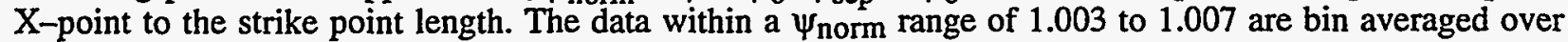
$\mathrm{L}_{\text {norm }}=0.125$. To obtain better statistics data from three repeat discharges have been averaged together. Langmuir probe data at the strike plate and core Thomson scattering and CER data at the midplane at a $\psi_{\text {norm }}=1.005$ have been chosen for comparison to the model. The error bars shown in the Thomson scattering data are representative of the scatter in the data over which the averaging was done, and are much larger than 
the error in each Thomson scattering data point. This scatter is typical of divertor and SOL data during ELMing discharges. 6

As can be seen, the modeling code does a reasonably good job of matching the data. Notable is that the rapid drop in the electron density away from the plate predicted by the code, due to the recycling neutral source, matches reasonably well with the Langmuir probe measurements at the plate and the Thomson scattering points just above the plate. Table 2 compares the plasma data at $\Psi_{\text {norm }}=$ 1.005 at the midplane with the code predictions. Ion temperatures significantly higher than the electron temperatures are routinely seen in the model and in the data. To match the CER data a distribution of power flux favoring the ion channel was required in the code (Table 1). In the low power case, it was not possible to increase the midplane ion temperature up to the measured value while keeping the total power flux at a reasonable level and maintaining agreement with the divertor plasma density.

Figure 2 shows a comparison of the measured line emissivities to simulated emissivities for both the high and low power beam injection cases and for two neutral carbon source models: 1) sputtering only, dashed line, and 2) sputtering plus carbon neutral injection along a $5 \mathrm{~cm}$ length of the divertor leg above the strike plate, solid line. The data is plotted as the measured line emissivities versus $\Delta R_{\text {sep }}$, the distance between the radial location of the outer strike point and the position of the viewing chord on the divertor floor, and similarly for the model. The agreement for line emission intensities of CII, III and IV and the spatial distributions of CIV is reasonable. The model indicates that the dominate source for CII and CIII line emission is the sputtered carbon. However for CIV, line emission from carbon that exists well above the strike plate contributes strongly to the modeled signals, resulting in a significant difference between the two cases with and without neutral carbon injection.
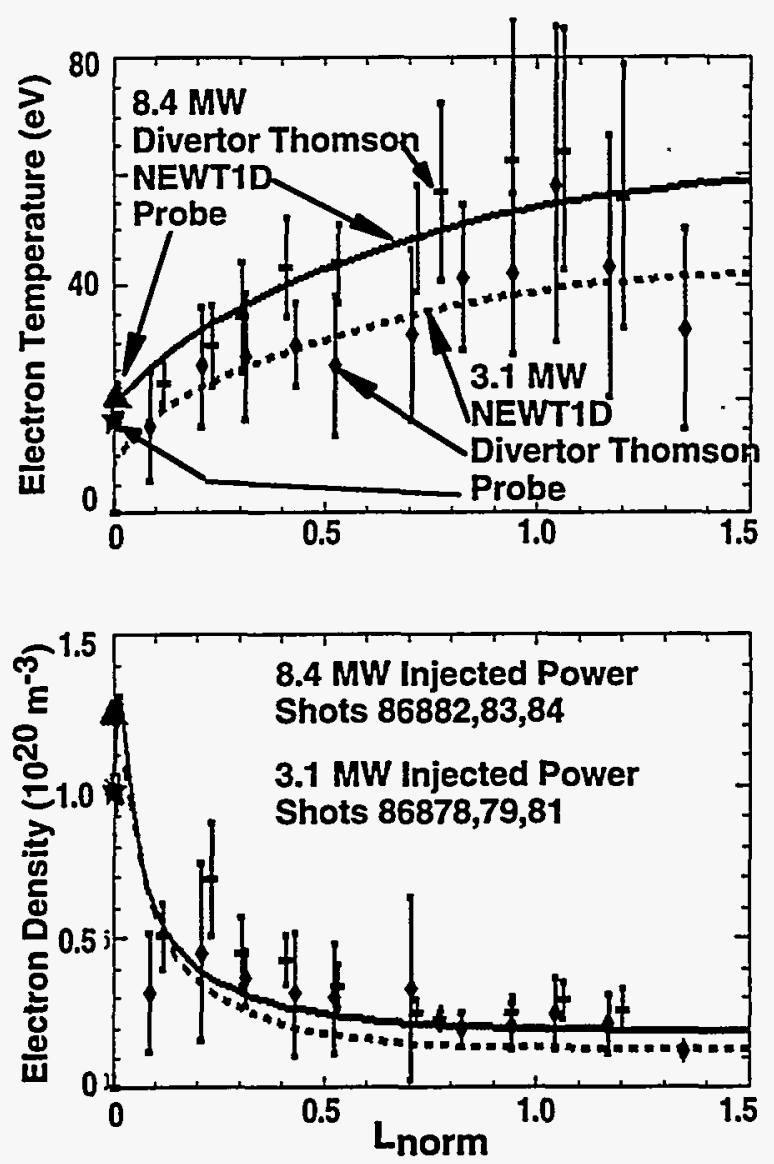

Fig. 1. Comparison of the NEWT1D simulations to the measured plasma electron temperature and density along the divertor leg from the strike plate to near the $\mathrm{X}$-point. The data are plotted as a function of the normalized poloidal distance, defined in the text. The crosses (8.4 MW) and diamonds (3.1 MW) with error bars are the Thomson scattering data, as describe in the text. Langmuir probe data are plotted at $\mathrm{L}_{\text {norm }}=0$.

\begin{tabular}{lcccccc}
\hline Table 2 & \multicolumn{3}{c}{$3.4 \mathrm{MW}$} & \multicolumn{4}{c}{$3.1 \mathrm{MW}$} \\
\hline & $\mathrm{n}_{\mathrm{e}}$ & $\mathrm{T}_{\mathrm{e}}$ & $\mathrm{T}_{\mathrm{i}}$ & $\mathrm{n}_{\mathrm{e}}$ & $\mathrm{T}_{\mathrm{e}}$ & $\mathrm{T}_{\mathrm{i}}$ \\
& $10^{19} \mathrm{~m}^{-3}$ & $\mathrm{eV}$ & $\mathrm{eV}$ & $10^{19} \mathrm{~m}^{-3}$ & $\mathrm{eV}$ & $\mathrm{eV}$ \\
\cline { 2 - 7 } Simulation & 1.9 & 66 & 233 & 1.3 & 47 & 180 \\
Plasma Data & $1.7(0.16)$ & $65(6)$ & $230(50)$ & $1.0(0.1)$ & $40(4)$ & $240(50)$ \\
\hline
\end{tabular}

\section{Discussion}

NEWTID code gives remarkably good agreement with the measured divertor plasma parameters in two ELMing $\mathrm{H}$-mode plasmas at two different heating powers using input parameters consistant with the operating conditions. The measured electron density and temperature near the strike plate, and their poloidal gradients, as well as the ion temperature at the midplane, are in good agreement with the simulations.

The measured CII and CIII spatial distributions are broader than the model predictions, which is not surprising since the these ionization states should be dominant in the colder, less dense outer regions of the divertor and SOL which are not modeled. To accurately model these regions requires a $2 \mathrm{D}$ code. The spatial 
distribution of the CIV emission predicted by the code, which is likely to come predominately from the hot, dense part of the SOL nearest the sepratrix, matches the data reasonably well.

In both the high and low power simulations, considerable sputtering from the divertor strike plate results in very little carbon transported to the midplane. To achieve a level of CIV line emission observed in the experiment, neutral carbon must be injected into the flux tube well above the recycling zone. Similar conclusions were drawn by Matthews et. al. ${ }^{8}$ and Krieger et. al. 9 using Monte Carlo techniques for modeling carbon spectroscopy on JET and ASDEX-Upgrade. Stangeby and Elder ${ }^{10}$ provide an in-depth discussion of the transport physics in the recyling zone that leads to the entrainment of sputtered impurities. Near the plate in the strong recycling zone, the strong flow of the primary ion
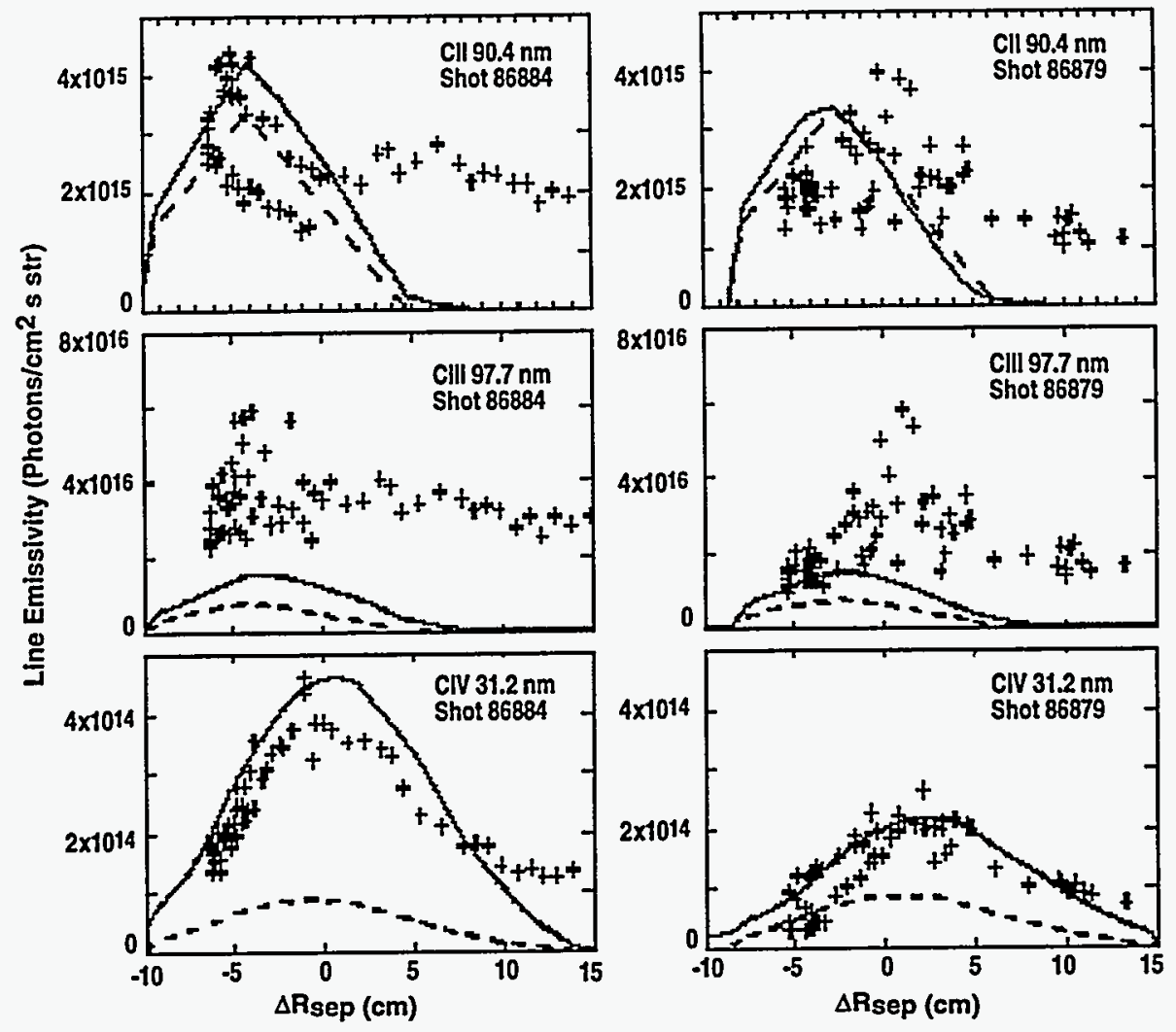

Fig. 2. Comparison of the simulated and measured line emissivities for $\mathrm{CII}$, III, and IV VUV lines for the 8.4 MW and 3.1 MW ELMing $\mathrm{H}$-modes. Crosses are the measured values. The solid (dashed) lines are for the simulation with (without) neutral carbon injection along a $5 \mathrm{~cm}$ poloidal length of the flux tube just above the strike plate. Both simulation cases include sputtering.

fluid toward the plate provides a strong frictional push on the impurity ions. If the sputtered neutrals are ionized before escaping the recycling zone, they are trapped at the plate. However, just above the plate the frictional force is significantly reduced and the ion temperature gradient force pushes the impurity ions toward the midplane. Stangeby and Elder refer to processes that lead to such neutrals as "deep injection" These results indicate the importance of neutral transport. More complete simulation of carbon in a tokamak plasma will require a more physically realistic model of neutral carbon (or hydrocarbon) generation.

${ }^{1}$ M.E. Fenstermacher, et al.,"Comprehensive 2D Measurements of Radiative Divertor Plasmas in DIII-D," Proc. of 12th Conf. Plasma Surface Interactions, St. Raphael, France 1996.

2S.I. Braginskii, Revof Plasma Physics, Vol 1. Ed. M.A. Leontovich, New York (1965).

3S. P. Hirshman, Phys Fluids 20, 589 (1997).

${ }^{4}$ R. A. Hulse, Nucl Techn/Fusion 3, 259 (1983).

5R.C. Isler, et al., "Spectroscpic Characterization of the DIII-D Divertor," to be submitted to Physics of Plasmas, 1996.

6R. Moyer, et. al., "The Role of Turbulent Transport in DIII-D Scrape-off Layer and Divertor Plamsas," Proc. of 12th Conf. Plasma Surface Interactions, St. Raphael, France 1996.

$7_{\text {R. W. Wood, this conference. }}$

${ }^{8}$ G.F. Matthews et. al., Nucl Mater 196-198, 374 (1992).

${ }^{9} \mathrm{~K}$. Krieger, et. al., Nucl Mater 220-222, 548 (1995).

10P. C. Stangeby and J.D. Elder, Nucl Fusion 35, 1391 (1995). 